\title{
Does Abalone Nacre Form by Heteroepitaxial Nucleation or by Growth through Mineral Bridges?
}

\author{
Tilman E. Schäffer, ${ }^{*}, \dagger$ Cristian I onescu-Zanetti, ${ }^{\dagger, \perp}$ Roger Proksch, ${ }^{\dagger}$ \\ Monika Fritz, ${ }^{\dagger, \ddagger}$ Deron A. Walters, ${ }^{\dagger}$ Nils Almqvist, ${ }^{\dagger, l l}$ Charlotte M. Zaremba, ${ }^{\S, \perp}$ \\ Angela M. Belcher ${ }^{\ddagger, \S}$ Bettye L. Smith,,,$\perp$ Galen D. Stucky, ${ }^{\S, \perp}$ \\ Daniel E. Morse, ${ }^{\neq, \perp}$ and Paul K. Hansmat, ${ }^{\dagger}$ \\ Department of Physics, University of California, Santa Barbara, California 93106; Marine \\ Biotechnology Center and Department of Molecular, Cellular and Devel opmental Biology, \\ University of California, Santa Barbara, California 93106; Department of Chemistry, \\ University of California, Santa Barbara, California 93106; Materials Research Laboratory, \\ University of California, Santa Barbara, California 93106; and Department of Physics, Lulea \\ University of Technol ogy, S-97187 Luleå, Sweden
}

Received August 12, 1996. Revised Manuscript Received J une 10, 1997

\begin{abstract}
We present experimental support for a model of abalone nacre growth that is based on mineral bridges between successive aragonite tablets rather than on heteroepitaxial nucleation. Interlamellar sheets of organic polymers delineate the aragonite tablets but allow the tablets to grow mineral bridges through pores in the sheets. Atomic force microscope images of interlamellar organic sheets from flat pearls made by $\mathrm{Hal}$ iotis rufescens (red abalone; marine gastropod mollusk) reveal a fibrous core and holes of 5-50 $\mathrm{nm}$ in diameter. Scanning ion conductance microscopy shows that these holes are actually pores through the interlamellar sheets. With the help of statistical analysis we can associate the pore-to-pore spacings in the interlamellar sheets with the observed offsets of successive nacre tablets. These results, supplemented by AFM, SEM, and TEM images, support and extend the model of biofabrication of gastropod nacre which is based on mineral bridges between the aragonite tablets.
\end{abstract}

\section{Introduction}

Biomineralization, the formation of microstructured mineralized composite materials by living organisms, is widespread in nature, producing a great diversity of high-performance composites. ${ }^{1}$ Although the mechanisms of biomineralization are not yet completely understood, it is hoped that they may provide new models for the fabrication of advanced material $\mathrm{s}^{2-5}$ and give new insights into the genetic control of biological structure. $^{6}$ Biomineralized composite materials typically contain only a few weight percent of proteins or other biopolymers, yet these macromolecules direct the assembly of elaborately structured composites with remarkable precision over scales ranging from the atomic to macroscopic. ${ }^{1,3-5,7-17}$

The nacre of molluscan shell and pearl is a microlaminate composite of highly oriented $\mathrm{CaCO}_{3}$ crystals

† Department of Physics, University of California.

₹ Marine Biotechnology Center, University of California.

$\S$ Department of Chemistry, University of California.

$\perp$ Materials Research Laboratory, University of California.

"Luleå University of Technology.

* Corresponding author: tilman @physics.ucsb.edu.

${ }^{\otimes}$ Abstract published in Advance ACS Abstracts, August 1, 1997.

(1) The Mechanisms of Biomineralization in Invertebrates and Plants; Watabe, N., Wilbur, K. M., Eds.; University of South Carolina Press: Columbia, SC, 1976; pp 283-308.

(2) Heuer, A. H.; Fink, D. J .; Laraia, V. J .; Arias, J . L.; Calvert, P. D.; Kendall, K.; Messing, G. L.; Blackwell, J .; Rieke, P. C.; Thompson, D. H.; Wheeler, A. P.; Veis, A.; Caplan, A. I. Science 1992, 255, 1098.

(3) Mann, S. In Inorganic Materials; Bruce, D. W., O'Hare, D., Eds.; Wiley: New York, 1992; p 237.

(4) Mann, S. Nature 1993, 365, 499.

(5) Mann, S.; Archibald, D. A.; Didymus, J. M.; Douglas, T.; Heywood, B. R.; Meldrum, F. C.; Reeves, N.J . Science 1993, 261, 1286.

(6) Morse, D. E.; Cariolou, M. A.; Stucky, G. D.; Zaremba, C. A.; Hansma, P. K. Mater. Res. Soc. Proc. 1993, 292, 59. (in the aragonite polymorph) and proteins, exhibiting exceptional regularity and mechanical strength. .,2,18-30 $^{-1}$ The interdigitating brickwork array of crystals and

(7) Liu, J .; Sarikaya, M.; Aksay, I. Mater. Res. Soc. Symp. Proc 1992, 255, 9.

(8) Sarikaya, M.; Aksay, I. A. Mater. Res. Soc. Symp. Proc. 1992, 255, 293.

(9) Humbert, R.; Sarikaya, M.; Aksay, I. A.; Crabb, J . W.; Furlong C. E. Mater. Res. Soc. Symp. Proc. 1994, 330, 89.

(10) Birchall, J . D. Trans. J . Br. Ceram. Soc. 1984, 83, 158.

(11) Weiner, S. S. Am. Zool. 1984, 24, 945.

(12) Cariolou, M. A.; Morse, D. E. J . Comput. Physiol. B 1988, 157, 717.

(13) Weiner, S.; Addadi, L. Trends Biochem. Sci. 1991, 16, 252.

(14) Addadi, L.; Weiner, S. Angew. Chem., Int. Ed. Engl. 1992, 31, 153.

(15) Biomineralization: Chemical and Biochemical Perspectives Mann, S.; Webb, J .; Williams, R. J. P., Eds.; VCH: New York, 1989; pp 541 .

(16) Moradian-Oldac, J F Frolow, F.; Addadi, L.; Weiner, S. Proc R. Soc. London B 1992, 247, 47.

(17) Albeck, S.; Aizenberg, J .; Addadi, L.; Weiner, S. J . Am. Chem. Soc. 1993, 115, 11691

(18) Wada, K. Bull. Natl. Pearl Res. Lab. 1961, 7, 703-828.

(19) Towe, K. M.; Hamilton, G. H. Calcif. TissueRes. 1968, 1, 306318.

(20) Mutvei, H. Stockholm Contr. Geol. 1969, 20, 1

(21) Erben, H. K. Biomineralization 1972, 4, 15.

(22) Erben, H. K. Biomineralization 1974, 7, 14.

(23) Nakahara, H. Venus J pn. J . Malac. 1979, 38, 205

(24) Nakahara, H.; Bevelander, G.; Kakei, M. VenusJ pn. J . Malac. $1982,41,33$

(25) Nakahara, H. In Biomineralization and Biological Metal Accumulation; Westbroek, P., de J ong, E. W., Eds.; Reidel: Dordrecht, 1983; p 225.

(26) Nakahara, H. In Mech. \& Phylogeny of Mineralization in Bio. Sys.; Sugan, S., Nakahara, H., Eds.; Springer-Verlag: Berlin, 1989; pp 343.

(27) Watabe, N. Prog. Cryst. Growth Characteristics 1981, 4, 99147.

(28) Weiner, S.; Talmon, Y.; Traub, W. Int. J . Biol. Macromol. 1983, 5,325 . 
organic polymer matrix (ca. $1-5 \%$ by weight) results in a fracture toughness that is $\sim 3000$ times greater than that of pure aragonite. ${ }^{31,32}$

We recently developed an experimentally tractable model system, in which "flat pearls" are produced by the shell-secreting tissue of Haliotis rufescens (the red abalone, a large marine snail or gastropod mollusk), which facilitates analysis of the biosynthesis of nacre and the underlying calcite on which the nacre is deposited. ${ }^{33-36}$ These studies and in vitro analyses of the roles of the proteins extracted from the mineralized composites have demonstrated that (a) a specialized nucleating sheet of protein governs the first nucleation of highly oriented crystals of cal cite to form a "primer", (b) differential secretion of two different families of soluble polyanionic proteins mediate the "genetic switch" controlling the transition from the synthesis of calcite to the production of aragonitic nacre, and (c) the soluble polyanionic proteins cooperatively direct the crystallization process, controlling polymorph selection and atomic lattice orientation. ${ }^{35}$ Control, however, of the higher-order structure of nacre that can extend over macroscopic distances for thousands of layers of interdigitated crystals requires an additional mechanism.

Several theories have been advanced to explain the biofabrication of this material, including (1) crystallization within preformed organic matrix compartments, ${ }^{23,37-39}$ (2) episodic or quantized secretion and subsequent crystallization, layer by layer, of interlamellar organic sheets and mineral crystals, 19,27 and (3) termination of crystal growth by poisoning ${ }^{40-42}$ or heteroepitaxial capping, ${ }^{6}$ followed by heteroepitaxial nucleation of the next crystal layer. These models all rely (implicitly or explicitly) on the proposition that each crystalline layer is nucleated on the interlamellar organic sheet. Our results support a different mechanism.

Abalone nacre may instead be formed by the continuous growth of aragonite crystals, from one layer to the next, through pores in the interlamellar organic sheets delineating the layers. In a comprehensive review article, though Watabe focused his discussion on the "generally accepted" model that the interlamellar sheets serve as the sites of aragonite crystal nucleation, he also mentioned an alternate possibility of growth through pores in the interlamellar sheets. ${ }^{27}$ This follows the

(29) J ackson, A. P.; Vincent, J . F. V.; Turner, R. M. Proc. R. Soc. London B 1988, 234, 415.

(30) Sarikaya, M. Microsc. Res. Techn. 1994, 27, 360-375.

(31) Currey, J. D. Proc. Roy. Soc. London 1977, 196, 443.

(32) Weiner, S. Crit. Rev. Biochem. 1986, 20, 365-380. 1328.

(33) Fritz, M.; Radmacher, M.; Gaub, H. E. Biophys. J . 1994, 66,

(34) Zaremba, C.; Belcher, A. M.; Fritz, M.; Li, Y.; Mann, S.; Hansma, P. K.; Morse, D. E.; Speck, J . S.; Stucky, G. D. Chem. Mater. 1996, 8, 676.

(35) Belcher, A. M.; Wu, X. H.; Christensen, R. J .; Hansma, P. K.; Stucky, G. D.; Morse, D. E. Nature 1996, 381, 56.

(36) Wada, K. In Profiles of J apanese Science and Scientists; Yukawa, H., Ed.; Kodansha: Tokyo, 1970; p 227.

(37) Wise, S. W. Science 1970, 167, 1486-1488.

(38) Uozumi, S.; Togo, Y. J . Fac. Sci. Hokkaido University Ser. IV 1975, 17, 153-172. 92.

(39) Bevelander, G. B.; Nakahara, H. Calc. Tiss. Res. 1969, 3, 84-

(40) Wilbur, K. M. In Chem. Zool.; Florkin, M., Scheer, B. T., Eds.; Academic Press: New York:, 1972; Vol. VII, pp 103-145.

(41) Watabe, N. J. Ultrastr. Res. 1965, 12, 351-370.

(42) Weiner, S.; Traub, W. In Structural aspects of recognition and assembly in biological marcomolecules; Balaban, M., Sussman, J . L., Traub, W., Yonath, A., Eds.; Balaban ISS: Rehovot, 1981; pp 467482. similar hypothesis of Mutvei ${ }^{43}$ and of Wada for bivalve nacre. ${ }^{44}$ Our results support this hypothesis.

Studies revealed that the tablets of nacre made by Haliotis are atomically coherent and unicrystalline from one layer to the next, ${ }^{45-48}$ providing the first experimental support for this hypothesis. Pores had been observed in interlamellar sheets that were fixed, decalcified and examined by electron microscopy, 19,20,24,25,49 but concerns that these might reflect fixation artifacts caused by shrinkage remained. Resolution of true structural details of this intricate and highly ordered material requires nanoscale imaging without fixation or use of organic solvents. We show in this paper that demineralization of flat pearls makes analysis of the remaining insoluble matrix possible with the atomic force microscope (AFM $)^{50-52}$ and with the scanning ion conductance mi croscope (SICM). ${ }^{53-55}$ The SICM images the ion conductance of a membrane thus revealing channels through the membrane. Since no metallic coating is required for either AFM or SICM, the samples are available for enzymatic and biochemical analyses of composition. Results presented in this paper confirm the existence of nanopores in the interlamellar organic sheets and show that the stochastic spacing of these pores is sufficient to account quantitatively for the observed lateral offset of aragonite tablets from one layer to the next, supporting the model of nacre growth by mineral bridge formation.

\section{Materials and Methods}

AFM imaging was done on interlamellar organic sheets from nacre. The samples were prepared as follows: "Flat pearls"34,36,56 were first produced on cover glasses $15 \mathrm{~mm}$ in diameter that were inserted between the Hal iotis rufescens mantle (the shell-secreting tissue) and the shell. In response to this insert, the mollusk sequentially deposited first calcite and then aragonitic nacre on the cover glass surface. ${ }^{34,35}$ The flat pearls were then removed and stored in sea water. Flat pearls were demineral ized in $10 \mathrm{~mL}$ of $10 \%$ acetic acid for $2-3$ days, leaving a soft patch of organic material that was still iridescent. The organic sheets were then placed on a glass slide and sectioned with a razor blade into small squares $(1 \mathrm{~mm} \times 1 \mathrm{~mm})$; these were then sonicated for $10 \mathrm{~min}$ in $1 \mathrm{~mL}$ of Hepes buffer $(5 \mathrm{mM}$

(43) Mutvei, H. SEM 1979, 2, 457-462.

(44) Wada, K. Biomineralization 1972, 4, 141-159.

(45) Wise, S. W. Ecolgae Geol. Helv. 1970, 63, 775-797.

(46) Manne, S.; Zaremba, C. M.; Giles, R.; Huggins, L.; Walters, D. A.; Belcher, A.; Morse, D. E.; Stucky, G. D.; Didymus, J . M.; Mann, S.; Hansma, P. K. Proc. Roy. Soc. Lond. B 1994, 256, 17.

(47) Feng, Q. L.; Su, X. W.; Cui, F. Z.; Li, H. D. Biomimetics 1995, 3, 159-169.

(48) Aizenberg, J .; Hanson, J .; Weiner, S.; Addadi, L., personal communication.

(49) Grégoire, C. In Chemical Zoology; V-VII; Florkin, M., Scheer, B. T., Eds.; Academic Press: New York, 1972; Vol. V-VII, pp 45102.

(50) Binnig, G.; Quate, C. F .; Gerber, C. Phys. Rev. Lett. 1986, 56, 930.

(51) Rugar, D.; Hansma, P. Phys. Today 1990, 43, 23-30.

(52) Drake, B.; Prater, C. B.; Weisenhorn, A. L.; Gould, S. A. C.; Albrecht, T. R.; Quate, C. F.; Cannell, D. S.; Hansma, H. G.; Hansma, P. K. Science 1989, 243, 1586-9.

(53) Hansma, P. K.; Drake, B.; Marti, O.; Gould, S. A. C.; Prater, C. B. Science 1989, 243, 641-643.

(54) Prater, C. B.; Hansma, P. K. Rev. Sci. Instrum. 1991, 62, 26348.

(55) Proksch, R.; Lal, R.; Hansma, P. K.; Morse, D. E.; Stucky, G. D. Biophys. J . 1996, 71, 2155.

(56) Fritz, M.; Belcher, A. M.; Radmacher, M.; Walters, D. A.; Hansma, P. K.; Stucky, G. D.; Morse, D. E.; Mann, S. Nature 1994, $371,49$. 


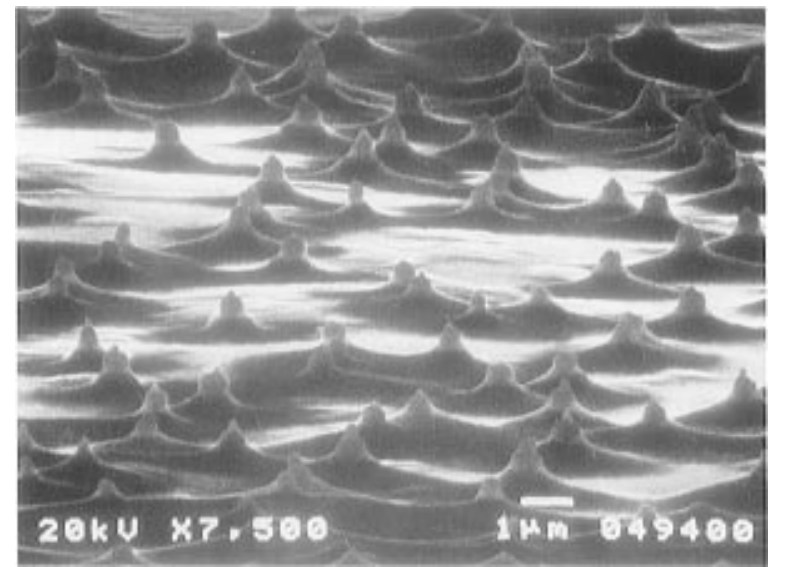

Figure 1. SEM micrograph of the growth surface (mantle facing surface) of Haliotis rufescens flat pearl nacre (oblique view). Single-crystal stacks of aragonite tablets are delineated by interlamellar organic sheets. Scale bar: $1 \mu \mathrm{m}$.

Hepes, pH 7.5) to separate consecutive sheets. A drop of the resulting suspension was placed on a freshly cleaved mica surface and gently dried with an air gun, leaving sheets adsorbed to the surface. The surface was then imaged in air with the AFM (Nanoscope III, Digital Instruments, Santa Barbara, CA) in contact mode. The resulting image was processed with Sharpen/U nsharp Mask in Photoshop (v.3.0, Adobe, Mountain View, CA) of radius $4 \mathrm{~nm}$ to bring out more clearly the surface features that were present in the raw data (Figure 2a). To obtain sheets treated with the enzymes, the sonicated sheets were centrifuged in an Eppendorf centrifuge at $12000 \mathrm{rpm}$ for $10 \mathrm{~min}$. The supernatant was removed and replaced by $1 \mathrm{~mL}$ of fresh Hepes buffer containing $200 \mu \mathrm{g} / \mathrm{mL}$ proteinase $\mathrm{K}$. The pellet was resuspended and sonicated in this solution for $10 \mathrm{~min}$. After incubation for $2 \mathrm{~h}$ at room temperature, the sample was centrifuged again (10 min at $12000 \mathrm{rpm}$ ) and the supernatant removed. The pellet was thoroughly washed in Hepes buffer, then incubated for $2 \mathrm{~h}$ at room temperature in $1 \mathrm{~mL}$ of $200 \mu \mathrm{g} / \mathrm{mL}$ collagenase and $200 \mu \mathrm{M} \mathrm{CaCl}_{2}$ in a Hepes buffer solution. The sample was then collected by centrifugation $(10 \mathrm{~min}$ at $12000 \mathrm{rpm})$, and the pellet sonicated in $1 \mathrm{~mL}$ of dei onized water. A drop of the resulting suspension was placed on a freshly cleaved mica surface and gently dried with an air gun. The surface was then imaged in air with the AFM in contact mode and the images processed as before (Figure $2 b, c$ ).

The interlamellar organic sheets were also investigated by scanning ion conductance microscopy (SICM) in tapping mode operation. ${ }^{53-55}$ SICM imaging was done on a sample prepared in a similar manner as the AFM samples: Flat pearls were demineralized, and the resulting thick organic patch containing the interlamellar organic sheets was rinsed and placed on the SICM sample holder (Figure 4). The patch was sealed tightly to a hollow Plexiglas chamber with a Teflon-coated magnetic washer. The solution was $100 \mathrm{mM} \mathrm{KCl}$. The Plexiglass chamber contained one of the two $\mathrm{Ag} / \mathrm{AgCl}$ electrodes. A bent, hollow micropipet with an aperture on the order of $100 \mathrm{~nm}$ in diameter was used as a cantilever and vibrated and scanned over the surface of the interlamellar sheets (Figure 5 ). The pipet was coated with $30 \mathrm{~nm}$ of gold to increase its reflectivity. The distance from the pipet to the surface was kept constant by detecting the vibrational amplitude of the
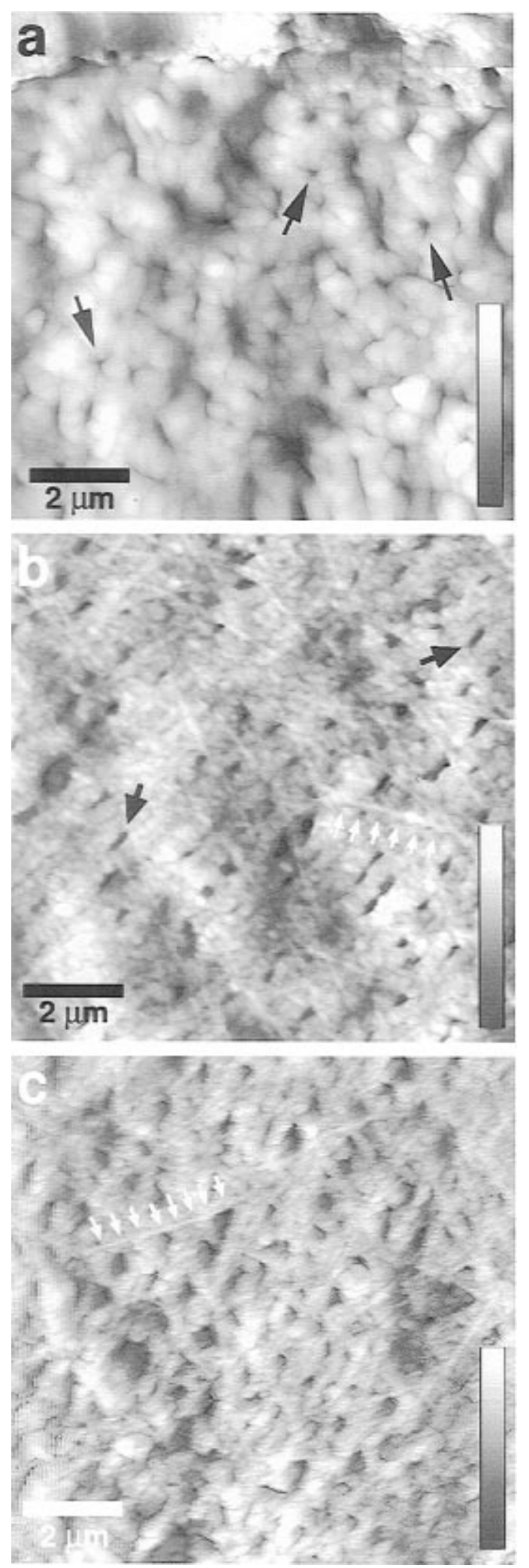

Figure 2. (a) AFM micrograph of unfixed demineralized interlamellar organic sheets from flat pearl nacre (dark regions are low and bright regi ons are high). The surface is putatively proteinaceous in nature and shows rounded features with an approximate diameter of $50 \mathrm{~nm}$ as well as pitlike structures (arrows). (b, c) AFM images of interlamellar organic sheets after enzymatic degradation with proteinases, revealing the core of the sheets. The core contains fibers (white arrows) and holes (black arrows) with spacings of $20-100 \mathrm{~nm}$. Scale bars: $2 \mu \mathrm{m}$. Height calibration bars: $0-60 \mathrm{~nm}$.

pipet with a conventional optical lever technique using feedback control similar to that in the tapping mode in AFM. Since the organic patch appeared to be as thick 


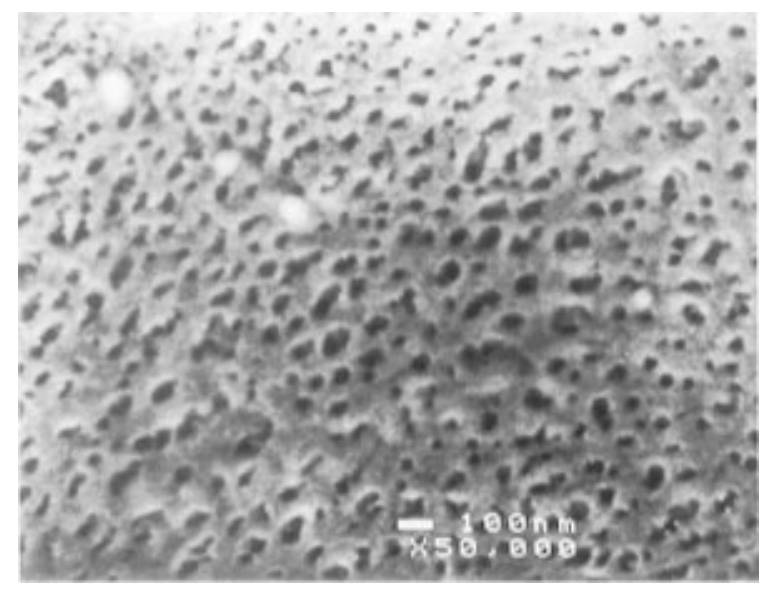

Figure 3. SEM micrograph of demineralized and fixed interlamellar organic sheets. The holes are spaced by 20-100 $\mathrm{nm}$, similarly to the holes of Figure 2. Scale bar: $100 \mathrm{~nm}$.

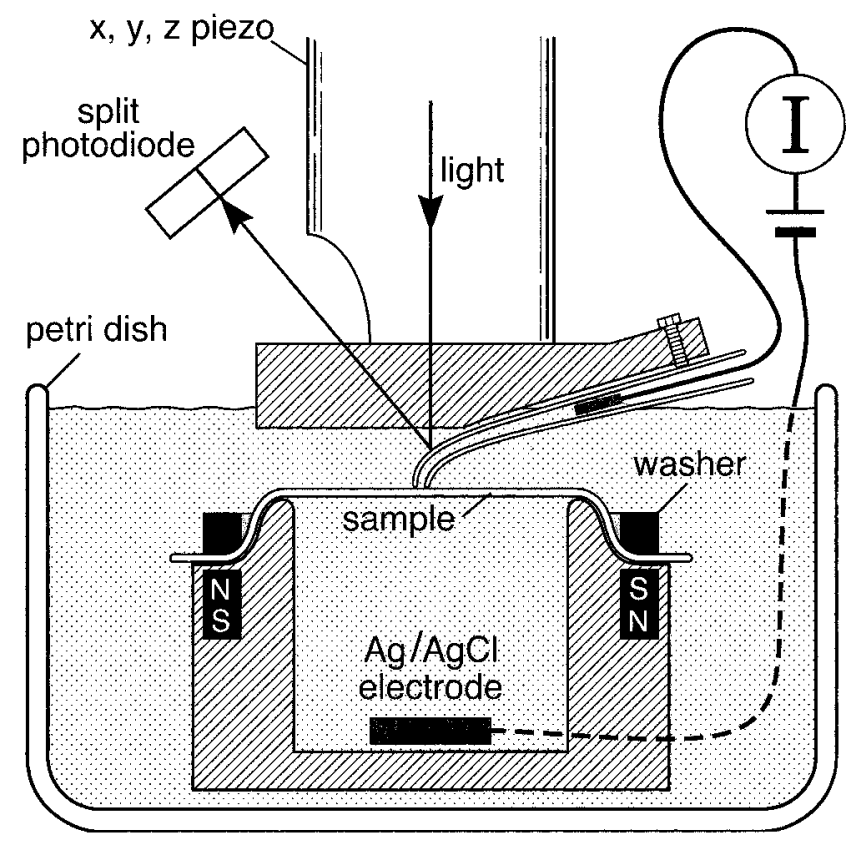

Figure 4. Combined scanning ion conductance microscope (SICM) and atomic force microscope (AFM). A pipet is scanned over the surface of the sample and the conductance through the sample is recorded. The deflection of the pipet, measured by a light beam reflected from the pipet, is held constant by a feedback loop.

and iridescent as the original flat pearl, we believe that the organic matrix was uncollapsed and that the interlamellar sheets retained their native separation of about $400 \mathrm{~nm}$.

Flat pearls used for SEM imaging (Figures 1 and 8) were stored in filtered sea water until immediately before imaging, rinsed with dei onized water, and blown dry. Some samples (Figure 1) were coated with $\sim 10 \mathrm{~nm}$ $\mathrm{Au}$ in an evaporator with sample-rotator attachment. These were imaged using secondary electron detection in a J SM-5300LV microscope (J EOL Ltd., Tokyo, J apan). Other samples (Figure 8) were imaged uncoated in this instrument using backscattered electron detection in environmental mode (low vacuum). Yet other flat pearl samples (Figure 13) were immersed in 5\% sodium hypochlorite (reagent grade) for $10 \mathrm{~min}$, rinsed with deionized water, and blotted dry with a paper tissue. These were imaged uncoated using secondary electron detection in a J SM-6300F microscope (J EOL) equipped with a cold cathode field-emission source.
Adult shells were demineralized and fixed in a $10 \%$ (v/v) acetic acid, 5\% formaldehyde (v/v using $37 \mathrm{wt} \%$ reagent grade) solution for 7 days with stirring, rinsed in several changes of dei onized water with stirring, and lyophilized in a Labconco freeze dry system. Pieces of the resulting organic matrix were mounted on conductive carbon tape, sputter-coated with $23 \mathrm{~nm} \mathrm{Au/Pd}$, and imaged at $3 \mathrm{kV}$ using the J SM-6300F microscope described previously (Figure 3 ).

TEM imaging (Figure 12) was done on a $50 \mathrm{~nm}$ thin section of flat pearl nacre fixed in $2 \%$ formaldehyde for $2 \mathrm{~h}$ and embedded in LR White "Hard" resin. The thin section was cut with a diamond knife and imaged with a J EOL 2000F TEM. The sample was unstained.

Cleaved nacre from the shell of an adult animal was sawed into pieces of well-defined shape. Therefore, top (toward mantle) and bottom (toward shell) surfaces were easy to identify. The nacre pieces were stored in 5\% sodium hypochlorite, saturated with respect to aragonite, for a few days. By this process, organic polymers were destroyed by oxidation while etching of the inorganic material was prevented by the presence of aragonite in solution. (When the sodium hypochl orite was not saturated with respect to aragonite, splitting of the pieces into flakes occurred, possibly the result of etching away inorganic connections between the tablets such as the proposed mineral bridges.) The bleached nacre was split into planar pieces by gently peeling away nacreous layers, glued onto stainless steel disks, rinsed with aragonite saturated deionized water and blown dry. The pieces were imaged in air (Figure 9) with the MultiMode Nanoscope III (Digital Instruments) in the tapping mode.

\section{Results and Discussion}

In the nacreous section of the gastropod mol lusk shell (Haliotis rufescens), aragonite tablets are enclosed by an organic matrix consisting of horizontal interlamellar organic sheets (Figure 1) and lateral walls. Previous TEM studies of fixed and stained vertical sections showed each interlamellar sheet to have a central, electron-dense core $\sim 10 \mathrm{~nm}$ thick, enclosed on top and bottom by granular layers (total thickness 40 nm). 20,24,26,32,49,57

In the AFM image of unfixed interlamellar sheets (Figure 2a) one can distinguish rounded features on the order of $30 \mathrm{~nm}$ with pitlike structures (arrows) in between. Fiberlike structures are not visible. Partial digestion with proteolytic enzymes (Figure $2 b, c)$ reveals the presence of holes (black arrows) $5-50 \mathrm{~nm}$ in diameter and of fiberlike structures (white arrows). The hole-to-hole spacing varies from 20 to $100 \mathrm{~nm}$, with an average value of about $50 \mathrm{~nm}$. This is in agreement with TEM studies of horizontal and vertical sections of fixed and demineralized nacre. ${ }^{20,24-26}$ Our AFM measurements reveal that the regions of low electron density observed in TEM are indeed holes in the organic sheets. The similarity in the spacings of the holes in Figure $2 b$ and the pitlike structures of Figure 2a suggests that the holes are present in both figures but that on the untreated surface they are harder to visualize because they are smaller in size. Another explanation might be that the upper layer of the organic sheet (Figure 2a), which is sensitive to proteinase $K$ digestion, is softer and thicker than the layer revealed after the proteolytic digestion (Figure 2b). A hole in a soft thick layer would 

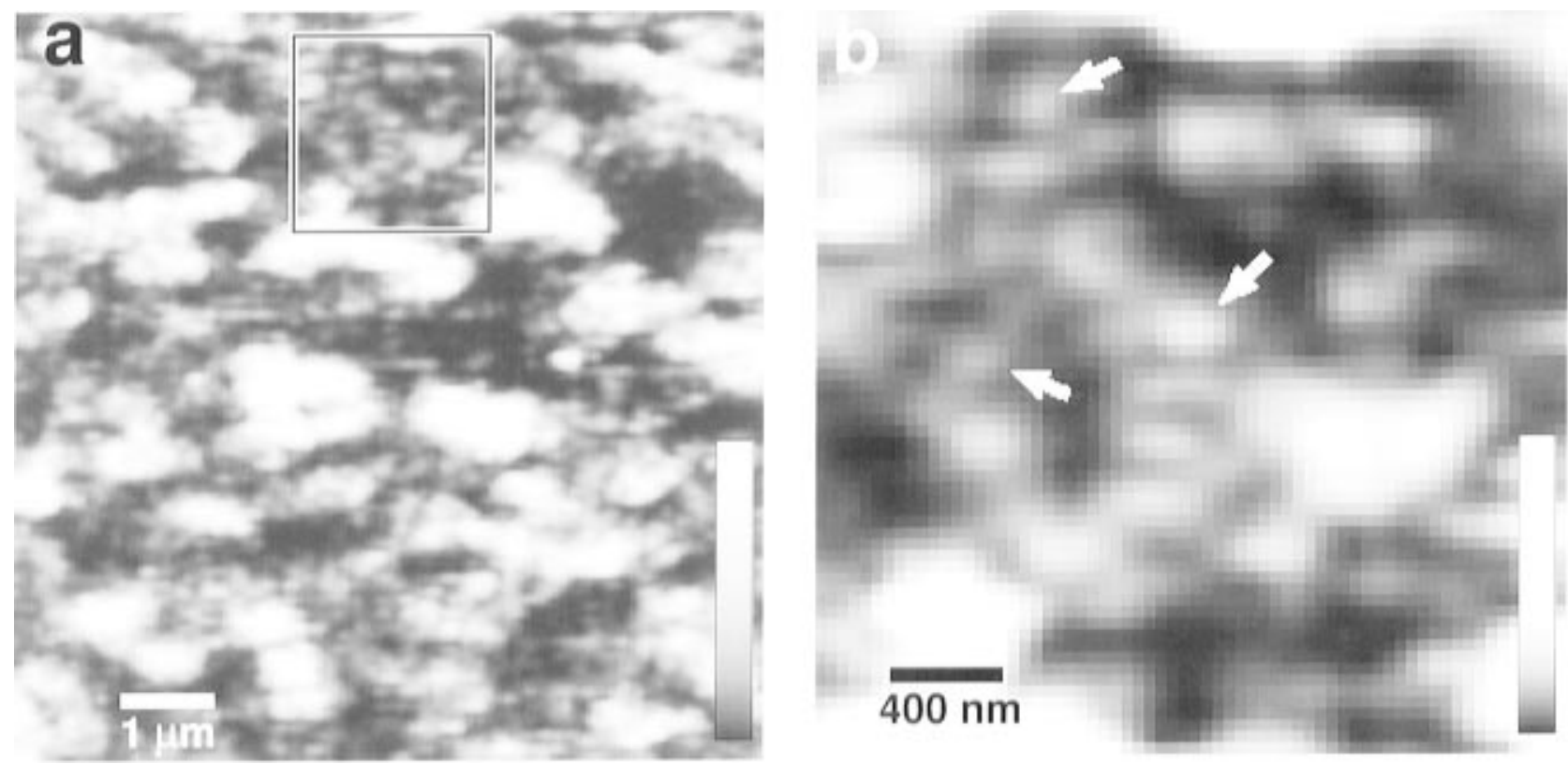

Figure 5. (a) SICM image of demineralized interlamellar organic sheets. Areas of low and high ion conductance can be seen, proving that the sheets are transparent for ions. Scale bar: $1 \mu \mathrm{m}$. Conductance calibration bar: 50-200 nS. (b) Expansion of the box in Figure 5a. Some areas with predominantly low ion conductance show small, distinct features that might be individual pores (arrows). Scale bar: $400 \mathrm{~nm}$. Conductance calibration bar: 50-200 nS.

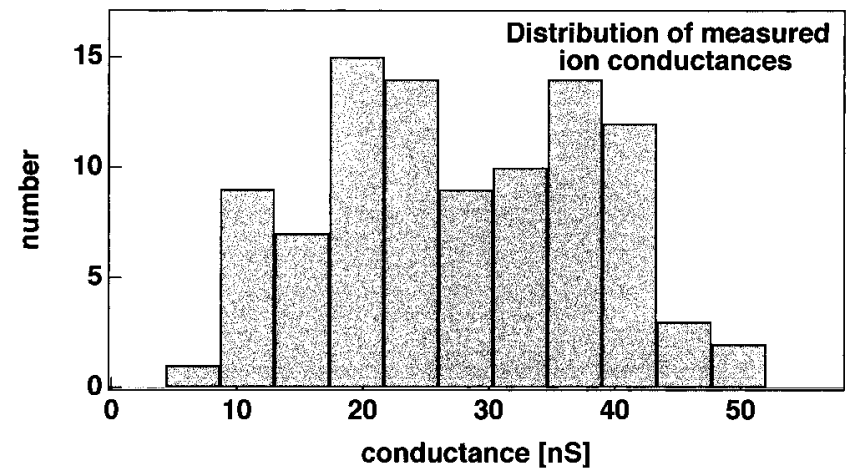

Figure 6. Histogram of measured ion conductances of the small distinct features in Figure 5. The ion conductances are distributed from 10 to $50 \mathrm{nS}$ and might represent individual pores.

appear smaller than a similarly sized hole in a hard thin layer because of tip-broadening. The holes in Figure $2 \mathrm{~b}$ are el ongated in one direction, possibly reflecting the orientation of the fibers or of the underlying crystal axes. The fibers, shown more clearly in Figure 2c, may correspond to the fibers thought by others to form the core of the interlamellar sheets. ${ }^{20,24,28,58}$ This core has been suggested, on the basis of el ectron micrographic and electron diffraction studies, to contain chitin or other structural polysaccharides. ${ }^{59,60}$ However, recent biochemical studies ${ }^{61}$ failed to detect $\mathrm{N}$-acetylglucosamine, the monomer of chitin, in hydrolysates of abal one nacre. Belcher, Howe, and Morse (unpublished) have shown that the insoluble matrix proteins associated with the interlamellar sheets are glycosylated. Results from experiments in progress in our laboratories show that at least one of the nacre-insoluble matrix proteins is collagenase sensitive. In Figure 2c, one can

(57) Addadi, L.; Weiner, S. Mol. Liq. Cryst. 1986, 134, 305-322.

(58) Grégoire, C. Bull. Inst. R. Sci. Nat. Belg. 1962, 38, 1-71.

(59) Peters, W. Comput. Biochem. Physiol. B 1972, 41, 541.

(60) Weiner, S. FEBS Lett. 1980, 111, 311-316.

(61) Keith, J .; Stockwell, S.; Remillard, K.; Kaplan, D. L.; Thannhauser, T.; Sherwood, R. Comparative Biochem. Physiol. 1993, 105, 487.

\section{Simple Theory}

(a) side view

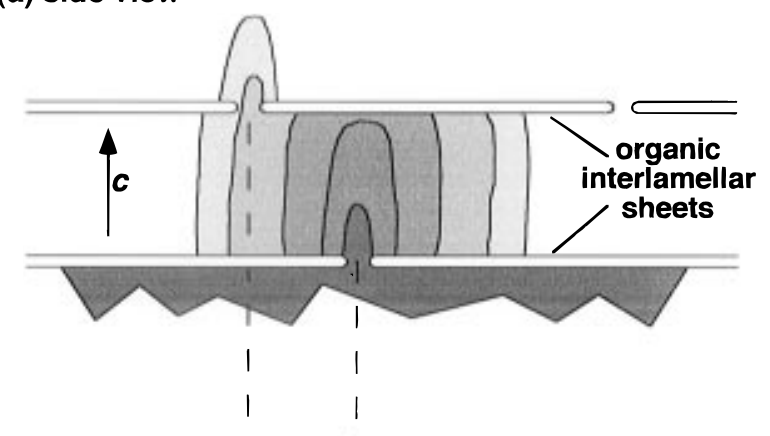

(b) top view

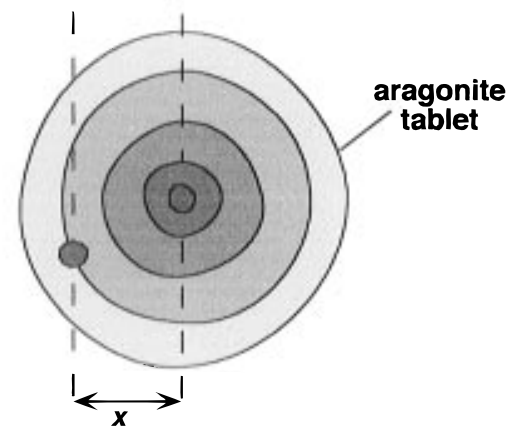

Figure 7. Schematic diagram of a simple model of nacre growth: (a) side view and (b) top view. In this model, aragonite nacre tablets grow through pores in the interlamellar organic sheets. The tablet offset seen in SEM micrographs (such as Figure 8) is the same as the pore offset seen in AFM and SICM images of the organic interlamellar sheets (such as Figures 2 and 5). The offset in the x-direction between the centers of two successive tablets is designated $x$.

clearly distinguish the presence of fibers about $10 \mathrm{~nm}$ in width. Although the aspect ratio of these fibers is similar to that ascribed to fibrils of chitin, $49,59,60$ these dimensions also are consistent with protein fibrils, and their composition remains uncertain. The average hole density seen in Figure $2 a-c$ is $97 \pm 35$ hol es $/ \mu \mathrm{m}^{2}$, where the error was estimated by comparing hole densities of different areas on the sample. In SEM micrographs of 


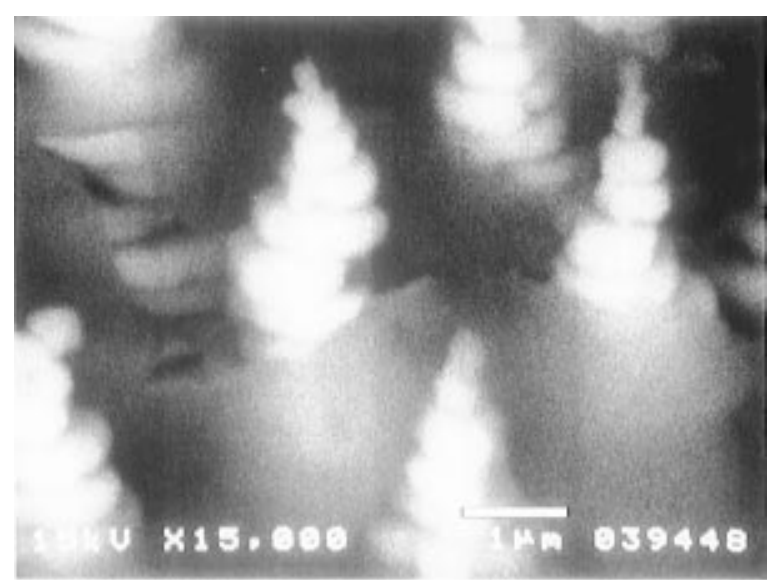

Figure 8. SEM micrograph of the growth front of flat pearl nacre. Growth direction is bottom to top. Notice the offset of the centers of successive aragonite tablets. Scale bar: $1 \mu \mathrm{m}$.

fixed interlamellar sheets (Figure 3), the holes are clearly visible. The average hole density is comparable with that from the AFM images.

SICM images of interlamellar organic sheets were taken to investigate the permeability of the sheets to ions (Figure 5). Areas of low and high ion conductance (Figure 5a) over the surface of the sheet can be seen. Some areas with predominantly low ion conductance show small, distinct features that might be individual pores (Figure $5 b$ ). The inner diameter of the probe and thus the lateral resolution was limited to the order of $100 \mathrm{~nm}$. Thus, large ion-conductance patches are presumably unresolved multiple pores. The equivalent electrical circuit of the SICM consists of a current source with a combination of resistors: the resistance of the SICM tip, in series with the resistance of the sample. The resistance of the sample consists of the resistance through the pore or pores over which the SICM tip is positioned, in parallel with a leakage resistance that is caused by an imperfect seal of the SICM tip with the sample, allowing ion flux through other channels in the sample. This leakage resistance results in a background to the SICM signal. In the following analysis we assume that this leakage resistance was constant at 50 nS (nanosiemens) over the SI CM image. This assumption is supported by the fact that the features seen in the SICM image did not reproduce in the simultaneous height image (data not shown).

By sampling a number of the smallest distinct features in Figure 5 that might represent indi vidual pores, we find that their ion conductances are distributed around $28 \pm 11 \mathrm{nS}$ (Figure 6). This result was independent of the particular selection of the features. From the theory of ionic channels $s^{62}$ we obtain for the diameter $\mathrm{d}$ of a pore in a membrane

$$
d=2 \sqrt{\frac{\rho l C}{\pi}}
$$

where $\rho$ is the resistivity of the solution, I is the thickness of the membrane and $\mathrm{C}$ is the ion conductance through the pore. Assuming ${ }^{63} \rho=80 \Omega \mathrm{cm}$ and $\mathrm{I}=30$ $\mathrm{nm}$, we obtain pore diameters of $15-40 \mathrm{~nm}$ with an average of $29 \mathrm{~nm}$. This is in agreement with the

(62) Hille, B. In Ionic Channels of Excitable Membranes; 2nd ed.; Sinauer Associates: Sunderland, MA, 1992.

(63) Bockris, J .; Reddy, A. Modern Electrochemistry; Plenum Press: New York, 1970. diameters of the holes observed in Figures 2 and 3, strongly suggesting that these holes are actually pores through the interlamellar sheets.

By dividing the integrated conductance (minus the 50 nS background) of the total scan area in Figure 5a by the average of the integrated conductances of single pores, we obtain a number that is representative of the number of pores in the image. With this procedure we account for the finite pi pet opening. We find that there are on the order of $5800 \pm 1200$ pores in the image, which corresponds to a pore density of $58 \pm 12$ pores/ $\mu \mathrm{m}^{2}$. This is of the same order of magnitude as the pore density observed in the AFM images (average of $97 \pm$ 35 pores $/ \mu \mathrm{m}^{2}$ ). The fact that fewer pores are observed in the SICM image could be explained by the possibility that not all holes seen in the AFM image are actually pores or that some are plugged pores. The similarities in the pore densities and pore diameters revealed independently by the AFM and SICM data supports the suggestion that the pores facilitate ion flux across the interlamellar sheets, as suggested by Towe and $\mathrm{Hamil}$ ton. ${ }^{19}$ It is al so likely that the pores facilitate diffusion of soluble anionic proteins necessary for control of tablet crystal growth. $35,64,65$

These observations are in accord with the hypothesis that the pores are responsible for controlling the position of the nucleation sites for the growth of tablets by allowing the aragonite to grow through them in the (vertical) c-axis direction (Figure 7). In this hypothesis, the aragonite grows through pores in the interlamellar sheets as a single crystal and thus maintains its crystallographic alignment not only in the c-axis as it grows from layer to layer, but also in the a- and b-axes. Such a crystallographic allignment was observed by Manne et al. while sequentially removing successive nacre tablets. ${ }^{46}$ Crystal axes coherence was confirmed for bivalve nacre by Feng et al. by showing that there is a domain structure of the orientation of the a-axis, which consists of 3-10 successi ve tablets from the same stack, with their a-axes parallel to each other. ${ }^{47}$ Similar observations for gastropod nacre were made by Aizenberg et al. using synchrotron X-ray diffraction to find crystal axes coherence in blocks of roughly 10-20 successive nacre tablets. ${ }^{48}$

In this hypothesis of the aragonite growing through pores in the interlamellar sheets, each newly nucleated tablet grows vertically toward the mantle, until it hits the next interlamellar sheet, where vertical growth is terminated. Then the tabl et grows laterally to form the new tablet. Note that the aragonite growth cones (Figure 8) are fairly steep. For a typical cone angle of about $40^{\circ}$, the vertical growth rate is more than 2 times the radial, lateral growth rate, indicating that a newly nucleated tablet grows fastest in the c-direction. Once the growing tablet hits a pore in the next interlamellar sheet above the tablet (mantle side), it grows through that pore as a mineral bridge, to nucleate a new tablet. The new tablet is offset laterally with respect to the underlying tablet, an offset readily observed in Figure 8. As the tablets grow laterally, more mineral bridges might form between the tablets (cf. Figure 13), which would allow the tablets to grow together in more than

(64) Walters, D. A.; Smith, B. L.; Belcher, A. M.; Paloczi, G. T.; Stucky, G. D.; Morse, D. E.; Hansma, P. K. Biophys. J . 1997, 72, 14251433.

(65) Falini, G.; Albeck, S.; Weiner, S.; Addadi, L. Science 1996, 271, 67-69. 

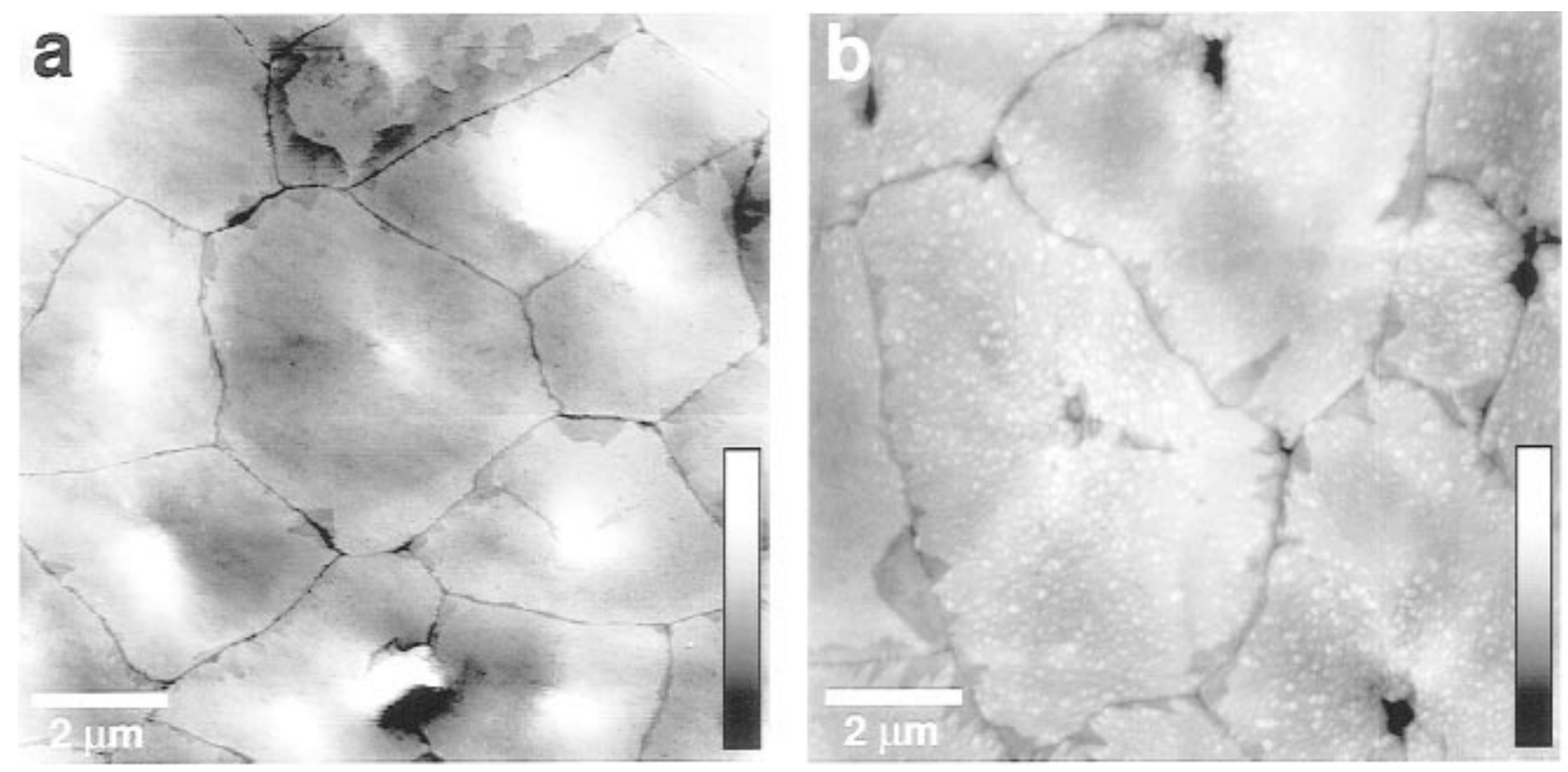

Figure 9. AFM micrographs (a) of the top surface (toward mantle) and (b) of the bottom surface (toward shell) of cleaved and bleached aragonite nacre. The central feature of each tablet constitutes a 80-150 nm high incline above the top nacre surface but is a $80-140 \mathrm{~nm}$ depression into the tablets on their bottom surface. Scale bars: $2 \mu \mathrm{m}$. Height calibration bars: $0-250 \mathrm{~nm}$.

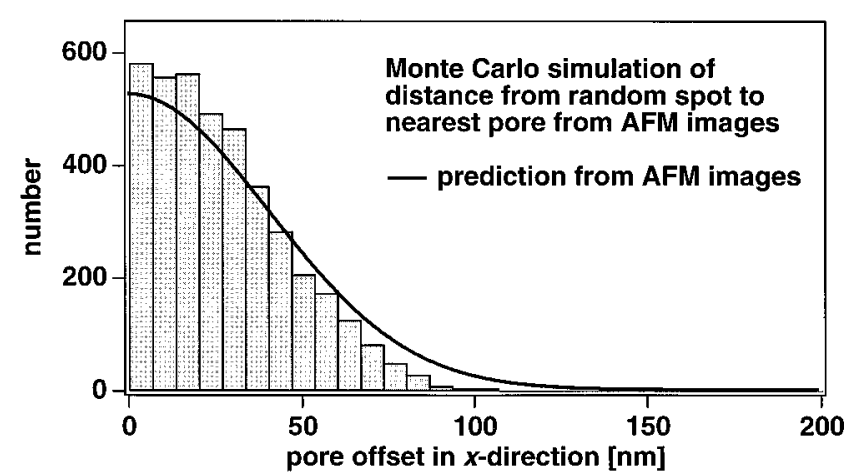

Figure 10. Histogram of the measured pore offsets in x-direction observed in AFM micrographs of interlamellar organic sheets, and a prediction based on a simple theory assuming a purely random pore distribution (solid line). The similarity of the measured and the predicted curve justifies the assumption.

one place (a similar observation on geological calcite was made by Hillner et al. ${ }^{66}$ ). It seems, however, that the first mineral bridge plays a particular role in nucleating the new tablet. This assumption is supported by AFM images of bleached and cleaved nacre (Figure 9). Clearly visible is a "central feature" on the tablets, observed before by others. ${ }^{20,46,67}$ This central feature, however, appears differently on the top (mantle) side of the tablets (Figure 9a) than on their bottom (shell) side (Figure 9b). A quantitative analysis shows that the central feature constitutes a 80-150 nm high incline above the top nacre surface but is a $80-140 \mathrm{~nm}$ depression into the tablets on the bottom surface. Therefore, it might be possible that the growing tablets push up the organic interlamellar sheet that is covering them, creating the central features observed in Figure 9 when they first hit the sheet (cf. Figure 1). It might be that the interlamellar sheet is originally very thin and has big pores but is in the process of getting thicker with smaller pores. Either way, this model of aragonite

(66) Hillner, P. E.; Manne, S.; Gratz, A. J .; Hansma, P. K. Ultramicroscopy 1992, 42-44, pt. B, 1387-93.

(67) Mutvei, H. Zool. Scr. 1978, 7, 287-296.

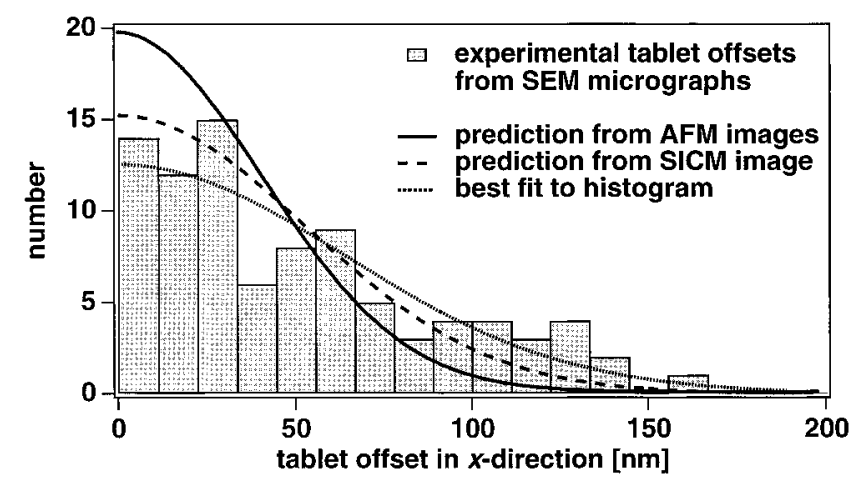

Figure 11. Comparison of experimentally measured tablet offsets from SEM micrographs such as Figure 8 to predictions based on pore densities from AFM images (solid line) and from SICM images (dashed line). The dotted line is a fit of the histogram to our simple theory with the pore density as the adjustable parameter, but the predictions have no adjustable parameters. The similarity of observed tablet offsets to the predicting curves suggests that there is a correl ation between the pores in the interlamellar sheets and the tablet offsets, supporting the model that the aragonite tablets grow through pores in the interlamellar sheets.

crystals growing through pores in the organic interlamellar sheets can account for the observed coherence of atomic lattice orientation of the crystals of vertically successive tablets in the nacreous layer of abalone shell. Further, previous studies ${ }^{46}$ showed that when the surface tablet layer is etched with $\mathrm{HCl}$, individual plates come off in one piece to reveal the underlying tablets, suggesting that there may be thin inorganic links such as mineral bridges between the tablets that will be etched first. We note, however, that there might be other nucleating mechanisms next to mineral bridges.

One prediction of this model, in which aragonite grows through pores from one tablet to the next, is that the offset of successive tablets is due to the offset of pores in the interlamellar sheets. If we assume that the pores are randomly distributed in the organic sheet with a certain average pore density, $n$, and that pores do not line up in successive sheets, then the radius, $r$, to which a tablet has to grow laterally until it hits the 


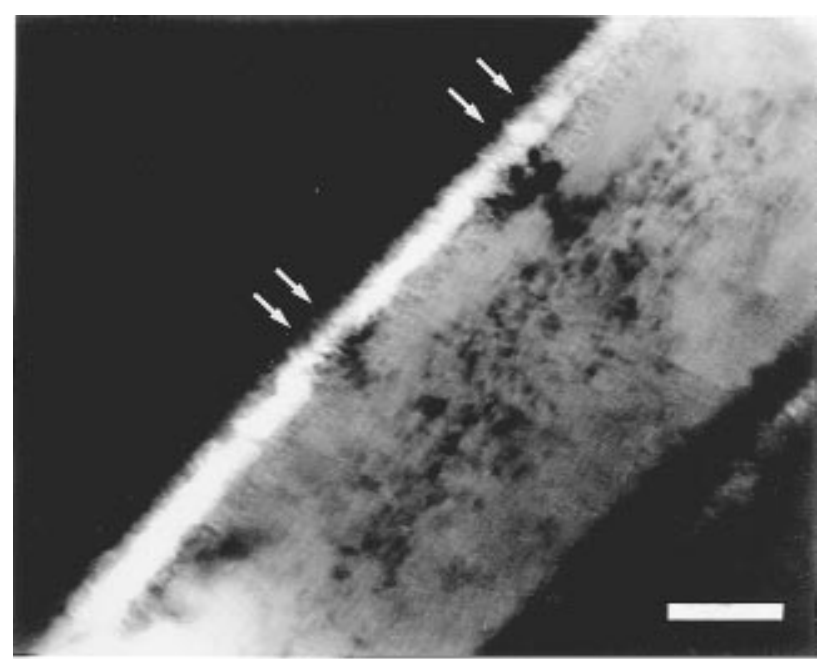

Figure 12. TEM micrograph of the region between two individual nacre tablets (one gray and one black). Only inorganic material is imaged. Gray bridges go through the bright region from one nacre plate to the other (arrows) and might be the proposed mineral bridges. Scale bar: $100 \mathrm{~nm}$.

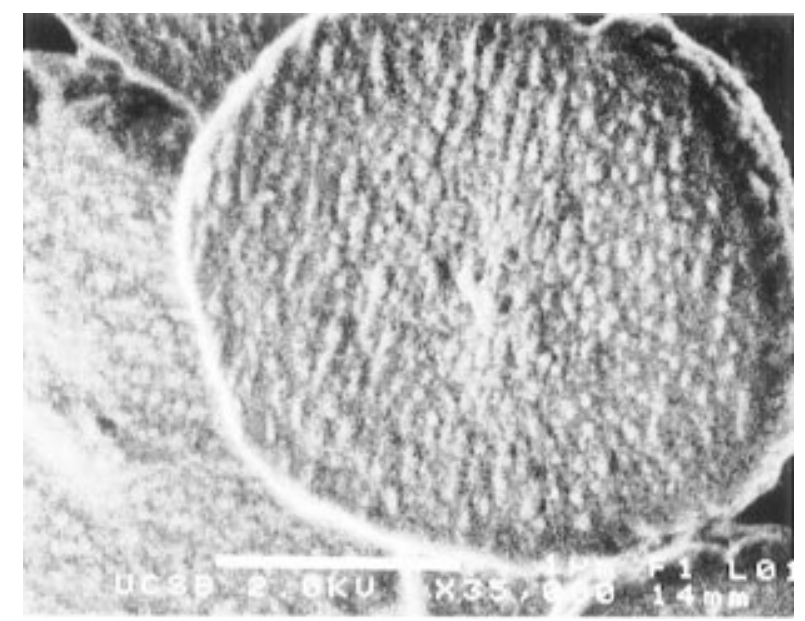

Figure 13. SEM micrograph of bleached aragonite nacre tablets. Many protrusions are visible, spaced by $40-90 \mathrm{~nm}$. The protrusions are elongated in one direction, in the plane of the tablet, similarly to the holes in the AFM images of Figure 2. The protrusions may be mineral growth into the pores of the interlamellar sheets which cover the tablets in the nacre. Scale bar: $1 \mu \mathrm{m}$.

first pore is a statistical distribution of functional form:

$$
p(r) d r=n e^{-\pi n r^{2}} \cdot 2 \pi r d r
$$

where $p(r) d r$ is the probability to find the closest pore within $r$ and $r+\delta r$. The radius, $r$, is the offset from the center of one tablet to the next. Therefore, assuming a uniform radial tablet growth rate, we expect the average radial tablet offset to be:

$$
\langle r\rangle=\int_{0}^{\infty} r p(r) d r=1 /(2 \sqrt{n})
$$

The average hole density from AFM images of interlamellar sheets (Figure 2 ) was $n=97 \pm 35$ holes $/ \mu \mathrm{m}^{2}$. With eq 3 we obtain an average radial offset $\langle r\rangle=$ $1 /(2 \sqrt{97})=51 \pm 9 \mathrm{~nm}$. From the assumption that the pores are randomly distributed it can be shown from the theory that the expected average radial tabl et offset equals the average pore-to-pore spacing between nearest-neighbor pores, thus yielding an average pore-topore spacing of $51 \pm 9 \mathrm{~nm}$. To compare the distribution of the tablet offsets expected from this calculation with the observed tablet offsets from Figure 8, we need to consider only the tablet offsets projected onto the x-axis, since Figure 8 is a vertical cross section of nacre and projects all distances into the image plane. Thus we calculate the projection of the statistical offset distribution, eq 2, onto the x-axis:

$$
\begin{aligned}
p_{x}(x) d x & =\left\{\int_{-\infty}^{\infty} n e^{-\pi n\left(x^{2}+y^{2}\right)} d y\right\} d x \\
& =\sqrt{n} e^{-\pi n x^{2}} d x
\end{aligned}
$$

$p_{x}(x) d x$ is the predicted probability to find the closest pore within $x$ and $x+d x$ and thus is the probability that the tablet offset lies within $x$ and $x+d x . p_{x}(x)$ is plotted as the solid line in Figure 10 using $n=97$ pores/ $\mu \mathrm{m}^{2}$. $\quad\left(\mathrm{p}_{\mathrm{x}}(\mathrm{x})\right.$ in Figure 10 is normalized with respect to the histogram area.) From eq 4 we obtain the moments of the distribution, $\langle x\rangle=0$ and

$$
\Delta \mathrm{x}_{\mathrm{RMS}}=\sqrt{\left\langle\mathrm{x}^{2}\right\rangle}=1 / \sqrt{2 \pi \mathrm{n}}=\sqrt{(2 / \pi)}\langle\mathrm{r}\rangle
$$

Equation 5 expresses the predicted RMS tablet offset in $x$-direction in terms of the average pore density, $n$, or in terms of the average radial tablet offset, $\langle r\rangle$. To verify that this prediction is valid for the observed distribution of pores in the interlamellar sheets, we need to establish that the pores are similarly distributed as the prediction of eq 4 . We performed a Monte Carlo simulation: In the sheets of Figure 2, we randomly picked points on the surface that represent the centers of the growing tablets below the sheets and measured the distance in the $x$-direction to the nearest pore in the sheets (where the next tablet will presumably be nucleated above the sheet). This measurement gives a distribution of distances representing the pore offsets in $\mathrm{x}$-direction that are the anticipated tablet offsets in $x$-direction and is displayed in the histogram of Figure 10. The distribution is similar to the prediction based on the density of pores in the sheets (solid line). For the anticipated RMS tablet offset in $\mathrm{x}$-direction we obtain $\Delta \mathrm{X}_{\mathrm{RMS}}=34 \mathrm{~nm}(\langle\mathrm{r}\rangle=43 \mathrm{~nm})$ for the measured distribution and $\Delta x_{R M S}=1 / \sqrt{2 \pi n}=41 \pm 7 \mathrm{~nm}(\langle\mathrm{r}\rangle=51$ $\pm 9 \mathrm{~nm})$ for the prediction based on $n=97 \pm 35$ pores/ $\mu \mathrm{m}^{2}$ (solid line in Figure 10). Therefore the measured anticipated average radial tablet offset is $17 \%$ smaller than what we would expect from a complete random pore distribution. This difference may be explained by correlations in pore locations due to the presence of fibers in the interlamellar organic sheets. On the basis of these results, however, assuming a random pore distribution seems to be sufficient to approximate the measured distribution of anticipated tablet offsets. This allows us to apply the statistics from eqs $2-4$ to the pores in the interlamellar organic sheets in the following analysis.

To test the model that the tablet offsets result from nucleation in pores in the interlamellar sheets, we measured offsets of successive tablets in SEM images (Figure 8). The histogram in Figure 11 is a compilation of all measurements taken independently by two researchers from different SEM images. We restricted the measurements to the top five tablets in each stack since we found larger offsets lower in the stacks, probably due to nonuniform tablet growth as the tablets become larger in size. The tablet offset is variable, from 0 to 
$150 \mathrm{~nm}$ in the $\mathrm{x}$-direction. The solid line is the prediction from Figure 10, based on the pore density in AFM images $\left(n=97\right.$ pores $\left./ \mu \mathrm{m}^{2}\right)$. The dashed line is the prediction with a pore density extracted from the SICM image $\left(n=58\right.$ pores $\left./ \mu \mathrm{m}^{2}\right)$. The histogram of observed values is similar in shape to the predicting curves. This suggests that there is a correlation between the pores in the interlamellar sheets and the observed tablet offsets, supporting the model that the aragonite tablets grow through pores in the interlamellar sheets. The dotted line is a fit of the data to eq 4 , with $\mathrm{n}$ as the free parameter, yielding $n=39 \pm 6$ pores $/ \mu \mathrm{m}^{2}$. The correspondence between the model and the observed values is better for the prediction based on the lower pore density from the SICM image, suggesting again that not all holes in the AFM images are actually pores. One unexplained feature is the presence of offsets greater than $100 \mathrm{~nm}$, which are predicted to be rarer than observed. This is, however, consistent with a requirement that a pore have a greater than average value of conductance to be suitable for mineral bridge formation. Using eq 5 , the average radial tablet offsets are $51 \pm 9$ and $66 \pm 7 \mathrm{~nm}$ for the predictions (Figure 11) based on AFM and SICM, respectively, and $80 \mathrm{~nm}$ from the fit of the measured offsets from SEM micrographs.

Another test of the model can be constructed from random-walk theory: If each tablet is offset laterally from the underlying one, we would expect a deviation of the tablet stack axis from the vertical. Specifically, if the offsets in $\mathrm{x}$-direction from one tablet to the next are governed by a distribution with a certain RMS offset, $\Delta \mathrm{X}_{\mathrm{RMS}}$, and each "step" (tablet offset) is independent from the others, then the distribution of offsets of tablets $m$ vertical layers apart from each other has an RMS offset that is independent of the offset distribution of two successive tablets. This RMS offset is

$$
\Delta \mathrm{X}_{\mathrm{RMS}}=\sqrt{\mathrm{m}} \Delta \mathrm{X}_{\mathrm{RMS}}
$$

In SEM micrographs (data not shown) we picked two tablets in the same stack that are separated by 50 layers and measured their offset in thex-direction. In this way we obtained 14 measurements, for different tablets in different stacks. This distribution had an RMS offset of $\Delta X_{\mathrm{RMS}}=474 \pm 260 \mathrm{~nm}$, yielding an RMS tablet offset of two successive tablets of $\Delta \mathrm{X}_{\mathrm{RMS}}=\Delta \mathrm{X}_{\mathrm{RMS}} / \sqrt{50}=67 \pm$ $37 \mathrm{~nm}$. From eq 5 , this corresponds to an average radial tablet offset of $\langle\mathrm{r}\rangle=84 \pm 46 \mathrm{~nm}$, which is in the same range as the average radial tablet offsets from Figure 11. This correspondence further supports the notion that the tablet offsets are due to the pore spacings in the sheets. It also supports the previous assumption that each tablet offset is independent from the previous one. We note, however, that from the observation of slight misalignments of the c-axes in successive tablets, ${ }^{68}$ an alternate explanation of the tablet offsets might possibly be constructed.

A previous analysis ${ }^{46}$ showed that even though successive tablets in the same stack are different in shape, their "central features" roughly line up with the underlying ones. A quantitative analysis (data not shown) reveals that they do not quite line up but are offset by an average of about $50-100 \mathrm{~nm}$ from one tablet to the

(68) Sarikaya, M.; Aksay, I. A. In Results and problems in cell differentiation in biopolymers; Case, S., Ed.; Springer-Verlag: Amsterdam, 1992; pp 1-25.

\section{OLD PARADIGM}
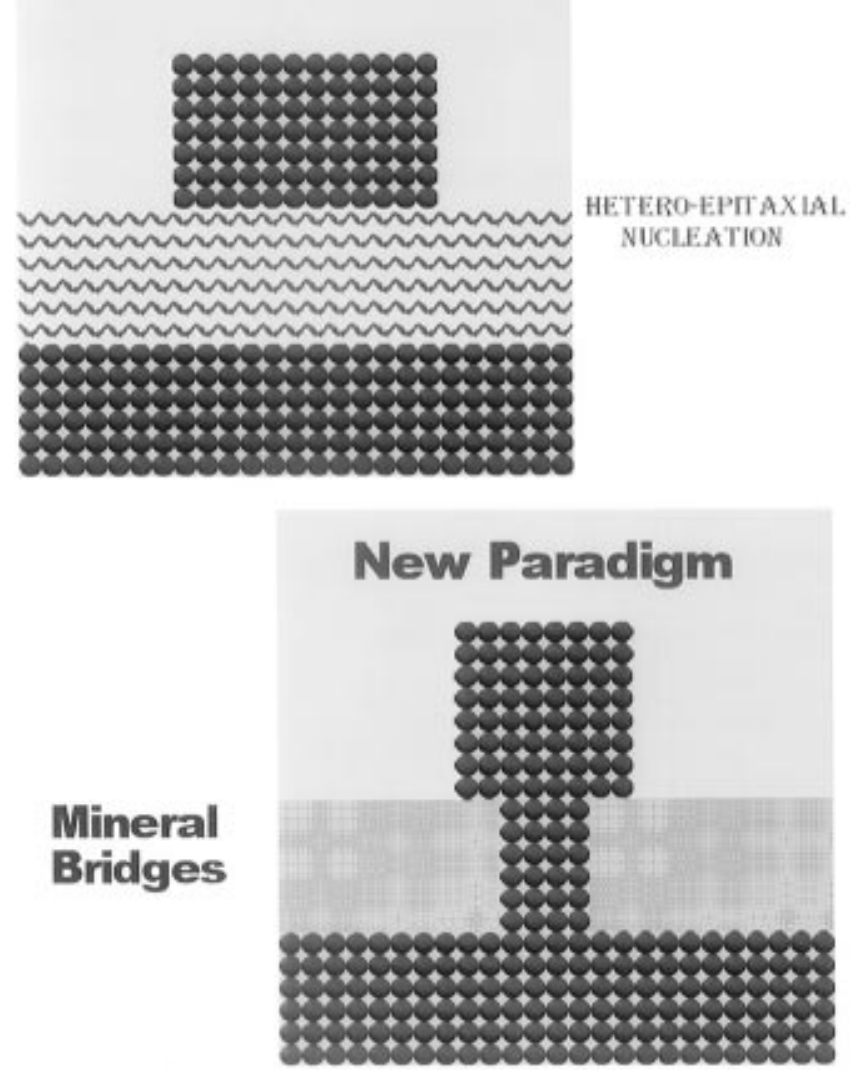

Figure 14. Two competing paradigms of nacre formation. In the old paradigm, the nacre tablets are heteroepitaxially nucleated on the surface of the interlamellar organic sheets. In the new paradigm, successive tablets grow from mineral bridges through the interlamellar sheets. The mineral bridges connect the tablets as a single crystal. As the tablets grow laterally, more mineral bridges might form between the tablets, allowing the tablets to grow together in more than one place.

next. Again, this is similar to the predicted tablet offsets, giving additional support for the mineral bridge hypothesis. The interdigitation observed between successive nacre tablets ${ }^{45,69}$ is on the order of microns, however, and cannot be explained by a $50-100 \mathrm{~nm}$ offset of the tablet centers. It is probably due to slightly uneven lateral tablet growth rates. Slightly uneven lateral growth rates are also suggested by Figure 9, since the tablet boundaries are slightly curved. This interdigitation may be one reason for the remarkable mechanical strength of nacre. ${ }^{70}$

Figure 12 is a TEM micrograph of the region (bright) between two individual nacre tablets (one gray and one black). Since the sample was unstained, only inorganic material is imaged. Note the gray bridges going through the bright region from one nacre plate to the other (arrows). These may be mineral bridges between the plates, but it is difficult to be certain of this assignment. While this TEM image does not conclusively prove the proposed model for nacre formation, it is consistent with this model. The putative mineral bridges in the image

(69) Nakahara, H. In Biomineralization and biological metal accumulation; Westbroek, P., de J ong, E. W., Eds.; D. Reidel Publishing Company: New York, 1983; pp 225-230.

(70) Gunnison, K. E.; Sarikaya, M.; Liu, J .; Aksay, I. A. In Hierarchically structured materials, Mater. Res. Soc. Symp. Proc; Aksay, I. A., Baer, E., Sarikaya, M., Tirrell, D. A., Eds.; Materials Research Society: Pittsburgh, 1992; Vol. 255, pp 171-183. 
have about the right spacing $(40-80 \mathrm{~nm})$. Their apparent thickness is somewhat smaller than the 15-40 $\mathrm{nm}$ we would expect based on the SICM measurements of ion conductance of the pores through which the mineral bridges grow. The apparent thickness in the image may, however, be an underestimate of the true thickness of the bridges; the core of the bridges is thicker and thus more easily imaged than the edges. Also, the thin section does probably not cut exactly through the center of a mineral bridge.

Further evidence for mineral bridges comes from SE M images of bleached aragonite tablets (Figure 13). Many protrusions are visible, spaced by $40-90 \mathrm{~nm}$. We count approximately $115 \pm 25$ protrusions $/ \mu \mathrm{m}^{2}$, which is similar to the average $97 \pm 35$ pores $/ \mu \mathrm{m}^{2}$ counted in the AFM images as described above. ${ }^{71}$ Also, the protrusions are el ongated in one direction, in the plane of the tablet, again similar to the AFM images (Figure 2). These results suggest that the protrusions may be mineral growth into the pores of the interlamellar sheets which cover the tablets in the nacre.

Figure 14 shows our primary conclusion in graphical form. Our data do not support the hypothesis that successive tablets are initiated by heteroepitaxial nucleation on the interlamellar sheets, as was the existing paradigm for many years. Rather, our data support a new paradigm: that successive tablets grow from mineral bridges through the interlamellar sheets, connecting the tablets together as a single crystal. This conclusion is consistent with Figure 1 , where mineral from the topmost layer prodrudes directly above the stack of tablets, instead of arbitrary locations.

\section{Conclusion}

The surface of demineralized interlamellar organic sheets of gastropod nacre (Haliotis rufescens) exhibits

(71) Due to this similarity it is unlikely that the holes observed in the interlamellar organic sheets are caused by artifacts of the sample preparation such as evolution of $\mathrm{CO}_{2}$ during dissolution. rounded features $30 \mathrm{~nm}$ in diameter. Proteinase $K$ and collagenase treatment reveals a fibrous core in the interlamellar sheets with hol es, 5-50 nm in diameter, spaced apart by $20-100 \mathrm{~nm}$, with an average spacing of about $50 \mathrm{~nm}$. This confirms previous TEM observations of the interlamellar sheets. SICM measurements show that these holes are actually pores through the sheets. A quantitative analysis proves that the poreto-pore spacing is sufficient to account for the lateral offset of successive nacre tablets from one layer to the next, supporting a model of coherent aragonite growth through the pores in the interlamellar sheets. Additional evidence for mineral bridges comes from a TEM image, where inorganic connections are observed between tablets. An SEM image of bleached nacre tablets shows protrusions on the surface that are spaced by 40$90 \mathrm{~nm}$, similar to the spacings of the holes in the interlamellar sheets $(20-100 \mathrm{~nm})$. Tablets from bleached nacre come off in one piece when etched, suggesting inorganic bridges between successive tablets. We now believe that abalone nacre grows not by heteroepitaxial nucleation, but rather by mineral bridge formation through interlamellar organic sheets.

Acknowledgment. We thank Neil $\mathrm{H}$. Thomson and Kerem Yaman for helpful discussions. This work was supported by a University of California Biotech grant (T.E.S.), the National Science F oundation under awards MCB-9202775 (M.F., D.A.W., G.D.S., D.E.M., P.K.H.) and DMR-9632716 (C.I.Z., C.M.B., B.L.S., G.D.S., D.E.M., P.K.H.), the Office of Naval Research under Award N00014-93-1-0584 (M.F., D.E.M., A.M.B., G.D.S., P.K.H.), the Army Research Office MURI Program under Award DAAH 04-96-1-0443 (G.D.S., P.K.H., D.E.M.) and the Swedish Research Council for Engineering Sciences under Grant 282-96-63. We thank Digital Instruments for AFM support. The first and second authors contributed equally to this work.

CM960429I 